\title{
Qualitative stomach cancer assessment by multi-slice computed tomography
}

\author{
Valoración cualitativa del cáncer de estómago mediante \\ tomografía computarizada multicapa
}

\author{
Gerardo Chacón ${ }^{1,2^{*}} \quad$ Johel E. Rodríguez ${ }^{1} \quad$ Valmore Bermúdez $^{1}$ \\ Miguel Vera ${ }^{3,4}$ Juan Diego Hernandez ${ }^{1} \quad$ Aldo Pardo $^{2} \quad$ Carlos Lameda $^{5}$ \\ Delia Madriz ${ }^{6} \quad$ Antonio José Bravo ${ }^{6}$
}

Recibido 9 de noviembre de 2018, aceptado 30 de octubre de 2019

Received: November 9, 2018 Accepted: October 30, 2019

\begin{abstract}
A theoretical framework based on the Borrmann classification and the Japanese gastric cancer classification is proposed in order to qualitatively assess the stomach cancer from the three-dimensional (3-D) images obtained using multi-slice computerized tomography (MSCT). The main goal of this paper is to demonstrate through visual inspection, the MSCT capacity to effectively reflect the morphopathological characteristics of the stomach adenocarcinoma types. The idea is to contrast the pathological theoretic characteristics with those that are possible to understand from MSCT images available in clinical datasets. This research corresponds to a study with a mixed approach (qualitative and quantitative), applied to a total of 46 images available for patients diagnosed, from the data collection included of the Cancer Genome Atlas Stomach Adenocarcinoma (TCGA-STAD). The conclusions are established from a comparative analysis based on the document review and direct observation, the product being a matrix of compliance with the specific qualities of the theoretical standards, in the visualization of images performed by the clinical specialist from the datasets. A total of 6210 slices from 46 MSCT explorations are visually inspected, and then visual characteristics are contrasted with respect to the theoretic characteristics obtained from the cancer classifications. These characteristics match into about $96 \%$ of images inspected. The approach effectiveness measured using the positive predictive value is about $96.50 \%$. The results of the images data also show a sensitivity of $97.83 \%$, and specificity of $98.27 \%$. MSCT is a precise imaging modality in the qualitative assessment of the staging of stomach cancer.
\end{abstract}

Keywords: Stomach cancer, adenocarcinoma, macroscopic assessment, Borrmann classification, Japanese classification, medical imaging, multi-slice computerized tomography.

1 Facultad de Ingeniería, Universidad Simón Bolívar, Cúcuta 540004, Colombia.

E-mail: j.chacon@unisimonbolivar.edu.co; jrodriguez116@unisimonbolivar.edu.co; v.bermudez@unisimonbolivar.edu.co; j.hernandezl@unisimonbolivar.edu.co

2 Grupo de Automatización y Control, Universidad de Pamplona, Cúcuta 540004 Colombia. E-mail: apardo13@hotmail.com

3 Facultad de Ciencias Básicas y Biomédicas, Universidad Simón Bolívar, Cúcuta 540004, Colombia. E-mail: m.avera@unisimonbolivar.edu.co

4 Grupo de Investigación en Procesamiento Computacional de Datos, Universidad de Los Andes-Táchira, San Cristóbal 5001, Venezuela.

5 Universidad Nacional Experimental Politécnica Antonio José de Sucre, Barquisimeto 3001, Venezuela. E-mail: lameda.carlos@gmail.com

6 Programa Calidad y Productividad Organizacional, Decanato de Investigación, Universidad Nacional Experimental del Táchira, San Cristóbal 5001, Venezuela. E-mail: dmadriz@unet.edu.ve; abravo@unet.edu.ve

* Autor de correspondencia: jrodriguez116@unisimonbolivar.edu.co 


\section{RESUMEN}

En el presente artículo se propone un marco teórico basado en la clasificación de Borrmann y la clasificación japonesa del cáncer gástrico para evaluar cualitativamente el cáncer a partir de imágenes tridimensionales (3-D) obtenidas mediante tomografía computarizada multicorte (MSCT). El objetivo es demostrar a través de la inspección visual, la capacidad de MSCT para reflejar efectivamente las características morfopatológicas de los tipos de adenocarcinoma de estómago. La idea es contrastar las características teóricas patológicas con aquellas que son posibles de comprender en las imágenes disponibles. Esta investigación corresponde a un estudio con un enfoque mixto (cualitativo y cuantitativo), aplicado a un total de 46 imágenes de pacientes diagnosticados, incluidos en el Atlas del Genoma del Cáncer (TCGA-STAD). Las conclusiones se establecen mediante un análisis comparativo basado en la revisión documental y observación directa, siendo el producto una matriz de cumplimiento de las cualidades específicas de los estándares teóricos, a partir de la visualización de imágenes realizadas por el especialista clínico. Se inspeccionaron visualmente un total de 6210 cortes de tomografía de 46 exploraciones de MSCT, y luego se contrastaron las características visuales patológicas con respecto a los criterios patológicos obtenidos de las clasificaciones de cáncer. Las características coinciden con aproximadamente el $96 \%$ de las imágenes inspeccionadas. La efectividad del enfoque medida usando el valor predictivo positivo es aproximadamente $96,50 \%$. Los resultados también muestran una sensibilidad de 97,83\% y especificidad de 99,18\%. MSCT es una modalidad de imagen precisa en la evaluación cualitativa de la estadificación del cáncer de estómago.

Palabras clave: Cáncer de estómago, adenocarcinoma, evaluación macroscópica, clasificación de Borrmann, clasificación japonesa, imágenes médicas, tomografía computarizada.

\section{INTRODUCTION}

Gastric adenocarcinoma classification follows the general perspectives of all types of cancer classification, namely: the histological (examination of tissue samples under a microscope) [1], the macroscopic (visualization of the morphology using imaging techniques) [2], the topographic (measurement of the changes of levels of the surface of the affected tissue) [3], by its biological behavior (intestinal and diffuse or expansive and infiltrating), by stage of the disease or stage of evolution. These individual classifications have been used by researchers in the worldwide, additionally, several clusters have been developed, of which, the most current, known and used in the research and clinical field, is the TNM Staging System. This staging system was developed and is maintained by the American Joint Committee on Cancer (AJCC) and the Union for International Cancer Control (UICC) [4-5].

The aim of the TNM system is the correct staging of the different tumors focused on clinical management, therapeutic decision (primary or adjuvant), monitoring, evaluation, forecast and/or prognosis, and unification of criteria for research projects and data transmission between research centers. This useful system allows to characterize tumors of any anatomical origin, and it is the support, in the diagnostic procedures, to conclude the prognoses estimated using others clinical approach [6]. The acronym TNM represents the completeness of the classification, which is shown in the Table 1.

However, this article is focused on the macroscopic method and the multi-slice computerized tomography (MSCT) imaging technique, whose theoretical reference scales are namely, the classifications of gastric cancer according to Borrmann [7] and the Japanase Gastric Cancer Association [8].

As mentioned, both classifications focus on the staging (stage of evolution) of the pathology, observable through images. In this sense, all tumors, after detection, are classified by staging [9], these being:

- Stage 0: It is denoted as cancer in situ. Groups of localized abnormal cells are present, which have not infiltrated the tissue. The cancer has not started to spread. Therefore, it is not a cancer in the strict sense, but a pre-cancer. This 
Table 1. Cancer Staging Scale TNM for gastric cancer [4].

\begin{tabular}{|c|c|c|c|}
\hline \multirow{2}{*}{ Acronym } & \multirow{2}{*}{ Meaning } & \multicolumn{2}{|r|}{ Classification } \\
\hline & & Code & Meaning \\
\hline \multirow{4}{*}{$\mathrm{T}$} & \multirow{4}{*}{ Primary tumor extensión } & $\mathrm{T} 1$ & Carcinoma in the mucosa and submucosa \\
\hline & & $\mathrm{T} 2$ & Carcinoma that extends to the subserosa \\
\hline & & $\mathrm{T} 3$ & Carcinoma infiltrating the adventitial or subserosal \\
\hline & & $\mathrm{T} 4$ & Carcinoma that compromises neighboring tissues \\
\hline \multirow{5}{*}{$\mathrm{N}$} & \multirow{5}{*}{ Presence of lymph nodes } & N0 & No involvement of lymph nodes \\
\hline & & $\mathrm{N} 1$ & Commitment of perigastric nodes \\
\hline & & $\mathrm{N} 2$ & Commitment of distant perigastric nodules \\
\hline & & $\mathrm{N} 3$ & Commitment of other intra-abdominal nodes \\
\hline & & $\mathrm{N} 4$ & Commitment of other nodes of extra-abdominal areas \\
\hline \multirow{2}{*}{ M } & \multirow{2}{*}{ Presence of metastases in distant organs } & M0 & Without distant metástasis \\
\hline & & M1 & Remote metástasis \\
\hline
\end{tabular}

stage corresponds with the best of situations, since by eliminating these cells, the cure is practically total.

- Stages I/II/III: The tumor is already cancer in the strict sense. Each tumor has its own description for each stage. The higher the stage, the greater the progress of the tumor, that is, the larger size and greater involvement of adjacent organs and structures. For example, a tumor in Stage I indicates that it only affects the organ and location where it originated. Neighboring organs are not affected.

- Stage IV: The tissue has a metastatic disease, that is, the abnormal cells are spread and the metastasis are disseminated to the rest of the body.

The utility of the staging is multiple; establishing an adequate stage of cancer allows a better dosing for the treatment and achieving the best chance of survival with less aggressive medical treatment [9]. In general, the staging requires as minimum to establish two classifications of the tumor. For example, if the patient undergoes surgery, the staging could be, one before starting the treatment and another after the surgery. A tumor can appear to be stage I before surgery, but after the operation, it is reclassified in stage II, which would indicate a worse prognosis.

In the next section, two macroscopic approaches are explained, according to their origin, their advances and their visual procedure for detecting of gastric adenocarcinoma.

\section{Borrmann Classification}

The first macroscopic classification of gastric cancer was proposed in 1926 by Borrmann [7]. Figure 1 shows the classification for the first four types of gastric cancer identified. This classification was updated to five types, and currently includes six types of gastric cancer.

Table 2 shows the nomenclature of Borrmann's current classification, from lowest to highest incidence. This classification allows to assess of early and advanced cancer. In this case, early cancer is denoted as Type 0 , which, although typed histologically, like the cancer Type V, cannot be assessed macroscopically from medical images. This classification is completely described as follows:
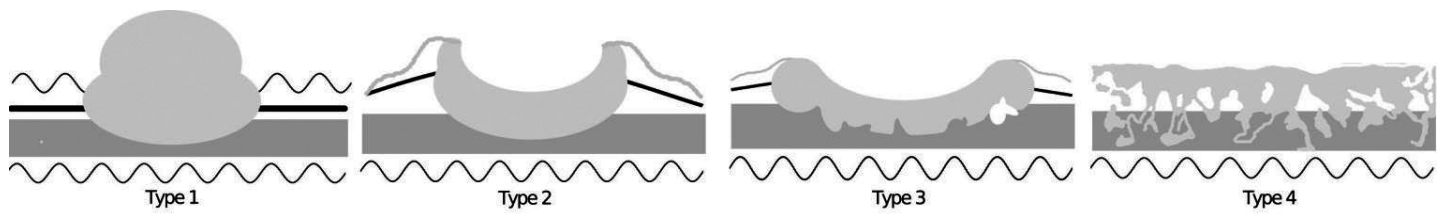

Figure 1. First Borrmann classification. 
Table 2. Extended Borrmann classification.

\begin{tabular}{|c|l|}
\hline Type & \multicolumn{1}{|c|}{ Name } \\
\hline 0 & Superficial tumor or depressed \\
\hline 1 & Polypoid \\
\hline 2 & Ulcerated circumscribed \\
\hline 3 & Infiltrating ulcerated \\
\hline 4 & Infiltrating linitis \\
\hline 5 & Anyone who does not fit in the previous types \\
\hline
\end{tabular}

- Type 0. Early cancer Superficial carcinoma with minimal depression or elevation. Cancers circumscribed, solitary and without ulceration, preferably located in fundus or greater curvature. These are the cancers with the best prognosis. They are the least frequent shape of presentation.

- Type 1. Advanced cancer of polyploid shape, with marginal elevation of parietal type and with well-defined contours. Of the advanced types of cancer assessed macroscopically, it constitutes the type of better prognosis. The most frequent shape, little infiltration, slow growth and late metastasis.

- Type 2. Ulcerated but circumscribed advanced cancer. They correspond to ulcerated cancers, partly with marginal elevation and partial diffuse dissemination. They are frequently located in the antro and lesser curvature.

- Type 3. Ulcerated cancer with carcinomatous infiltration.

- Type 4. Cancer with extended stomach infiltration. Ulceration is not an outstanding aspect. Tumors of great growth by the submucosa and subserosa. There are two types:

- Scirrhous: Infiltrating growth very rich in connective tissue.

- Brinton's plastic linitis: Malignant tumor.

- Type 5. Gastric cancers that can not be classified in other types.

In order to contribute the image visual assessment and the establishment of diagnostic conclusions, the precise characteristics of Borrmann classification of advanced cancer shown in Table 3 are considered in this research.

As a contribution, Kajitani [10] and Morson and Dawson [11] established other standards based on Borrmann's classification. Table 4 summarized the relationships between these three classifications.
Table 3. Precise characteristics of Borrmann for advanced cancer.

\begin{tabular}{|l|l|l|}
\hline Characteristic & Type 1 and 2 & Type 3 and 4 \\
\hline Boundaries & Well defined & No precise \\
\hline Location & Antro & More in depth \\
\hline Differentiation & Differentiated & Undifferentiated \\
\hline Acidity & Achlorhydria & Normal \\
\hline Forecast & Best & Worst \\
\hline Sex & More in men & More in women \\
\hline Age & More age & Less age \\
\hline Metastasis & Hematics & Lymphatics \\
\hline
\end{tabular}

Table 4. Relationship of the Borrmann classification with other subsequent classifications.

\begin{tabular}{|c|l|l|}
\hline Type & Kajitani $^{(\mathbf{1 0 )}}$ & Morson and Dawson \\
\hline 1 & Located & Nodular \\
\hline 2 & Intermediate & Ulcerating \\
\hline 3 & Infiltrative & Fungoid \\
\hline 4 & Infiltrative & Superficial plastic Linitis \\
\hline
\end{tabular}

In addition to the standards of current use such as TNM (previously section), there is the Japanese classification, which is analyzed in the following section, in which the description of cancers type 0 and type 5 by means medical imaging techniques are developed.

\section{Japanese Classification}

Inspired by the Borrmann classification, the Japanese Gastric Cancer Association (JGCA) established a set of rules for the classification of this pathology. This set of rules is known as Japanese Classification of Gastric Carcinoma (JCGC) [8]. The diagnostic, therapeutic and pathological rules are initially standardized by 1981 [10]. This classification is widely accepted and despite having undergone several revisions, the original structure remains. Recently, in 2010, the JGCA reviewed its classification and its treatment guide, the most relevant changes being the adoption of the classification proposed by the TNM (7th edition) and the definitions simplification of the different types of lymphadenectomy.

Additionally, the JGCA established as to the description of the macroscopic type for this pathology, that the tumors must have histologically treatment for their pathological classification. Also on this classification, by its macroscopic appearance, gastric 
cancer may be early or advanced. The early cancer is characterized as an "in situ" lesion, it is superficial and is shown as an irregular, indurated, elevated or depressed plaque, it is only confined in the mucous and/or submucosal layer. The type 1 tumors are typical of the early cancer with or without lymph node, the lesion can invade the mucous membrane and submucous. As the cancer deepens and invades structures such as the muscular or subserosa layer, advanced cancer or type 2 is referred to, and type 3 invades the serous layer. From the mucous surface view, the appearance of the macroscopic tumor is categorized into six types (see Table 2). Infiltration to adjacent structures is classified as type 4 . All this is shown in Figure 2.

Moreover, the Japanese classification coexists with that of Borrmann, extending it in terms of macroscopic types of type 0 early cancers. In this sense, the cancer type 0 is considered as superficial, the type $0-\mathrm{I}$ is typified as protruded, the type 0 -II is subclassified in superficial and elevated type, flat type and superficial and depressed type, type 0 -IIa, type 0 -IIb and type 0 -IIc, respectively. The type 0 -III corresponds with the excavated type. The Figure 3 and Table 5 show this classification.

Regarding the nomenclature shown in the Table 5, a lesion is considered protruded if it is greater than twice the mucous, and the lesion is considered as superficially elevated when it is no more than twice the mucous. As for the two classifications as a whole (Japanese and Borrmann), it is important to note that Japanese classification was previously used to diagnose cancer early and Borrmann for
Table 5. Typification of cancer type 0. Japanese classification.

\begin{tabular}{|c|c|c|l|}
\hline \multicolumn{2}{|c|}{ Type } & \multicolumn{2}{c|}{ Sub-type } \\
\hline Code & Name & Code & \multicolumn{1}{c|}{ Name } \\
\hline 0-I & Protruded & & \\
\hline \multirow{2}{*}{ 0-II } & \multirow{2}{*}{ Plane } & 0-IIa & Superficial and elevated \\
\cline { 3 - 4 } & & 0-IIb & Flat \\
\cline { 3 - 5 } & 0-IIc & Superficial and depressed \\
\hline 0-III & Excavated & & \\
\hline
\end{tabular}

advanced. Borrmann was used exclusively for advanced cancer that exceeds 3 or $4 \mathrm{~cm}$ in size, and invades the muscle wall, at least. However, the Borrmann classification is extended for use in detecting early cancer.

\section{Computed Tomography and Early Gastric Cancer Detection}

Computed tomography is a modality routinely used in clinical practice. This modality generates a set of two-dimensional images, each representing a slice that includes information about the anatomy of patient. 3-D images are obtained from radiological projections using reconstruction techniques. The projections are obtained by exposing the object to $\mathrm{X}$-ray radiation at different angles and by measuring the degree of absorption of the beam passing through the object [12].

Multi-Slice Computed Tomography (MSCT) is probably the most commonly used term to describe the latest developments in helical CT. This technology
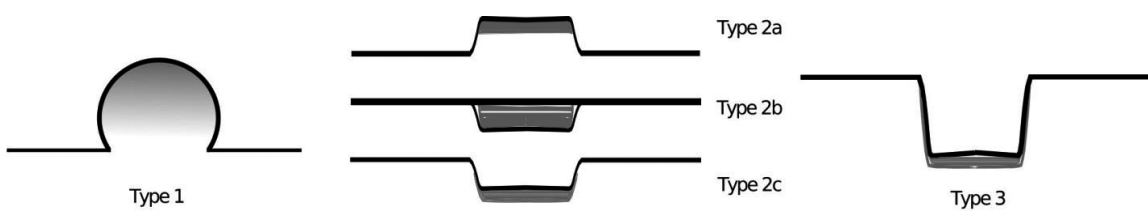

Figure 2. Japanese classification of early and advanced gastric cancer.

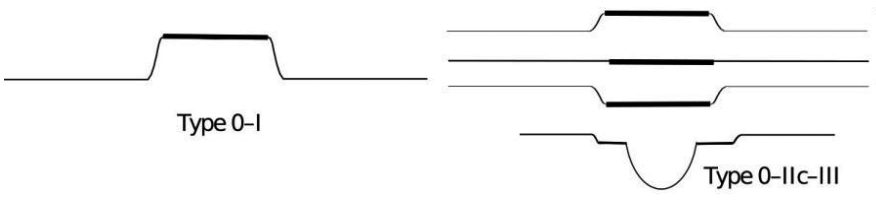

Type 0-lla

Type 0-IIb
Type 0-IIC

Figure 3. Japanese classification of cancer type 0. 
is based on the simultaneous acquisition of more than a single tomographic plane and it is closely related to the introduction of acquisition systems with multiple detectors [13]. The advances of MSCT have allowed developing tools useful for assessing of gastric cancer [14-16].

Early gastric cancer or cancer confined to the submucosa has been normally identified by means the endoscopic diagnosis [17]. However, in this research an approach based on MSCT image reconstruction in order to identify the gastric tumor is proposed. This approach initially considers the identification of the gastric tumor, from this identification, the location and the macroscopic characteristics of the adenocarcinoma are defined, then, such CT findings are compared with the prognosis of the endoscopy and of the histological diagnosis, and finally the degree of agreement is determined between assessment approaches.

In this paper, a procedure to assess of the morphopathological characteristics of the stomach neoplasms from computerized tomography images of the abdomen is proposed. The idea is focused to ensure efficiency, efficacy and effectiveness of the MSCT to reflect the main features of the malignant tumors types at stomach through the visual inspection guided by an expert physician.

\section{METHODS}

The expected result includes an evaluation process of the MSCT effectiveness (predictive capacity) for replicating the detection parameters, theoretically established, of types of gastric adenocarcinoma. The aim of this research is to compare the theoretical features inspected in the MSCT images with the parameters reported in the dataset considered in this work.

\section{Data Source}

The datasets considered in this research correspond with a part of the large National Cancer Institute (NCI)-funded initiative known as the Cancer Genome Atlas Stomach Adenocarcinoma (TCGA-STAD) [18]. The datasets connect cancer phenotypes to genotypes throughout medical images [19-20]. The datasets consider in this research correspond with 46 studies acquired using computed tomography medical imaging from 46 patients. The datasets contain 43908 images in 308 series. The images resolution the axial plane is $512 \times 512$ pixels, and the number of slices varies between 101 and 137 depending on the image, for example 512 x 512 x 101 voxels. The voxel size is $0.585938 \times 0.585938$ $\mathrm{x} 2.5 \mathrm{~mm}$. That is to say that quite small objects can be detected. Table 6 shows the original descriptive histological parameters of each case studied.

In Table 6, the descriptive histological parameters accompanied by an "a" were assessed during the autopsy; in the whole collection there are only two patients with metastasis at a distance from the tumor, they are indicated by the M1; and the parameters accompanied by NX and MX, indicate that regional lymph nodes cannot be evaluated and that metastasis cannot be evaluated, respectively.

\section{Phases of Research}

In general terms, the process for achieving the main objective is divided into two phases, explained according to the techniques used for analyzing the data.

The first stage consists of searching of the Borrmann and the Japanese classification theoretical features in the available datasets of MSCT images. The data collection technique is direct observation, which allows discovering and exposing the conditions of the investigated phenomena; that is, it helps the researcher to discern, inferring, hypothesizing and look for evidence [21]. In this sense, the sagittal and coronal reformatted images are reconstructed from cross sectional (axial) images obtained from the MSCT. The assessment consisted of the initial exploration of the magnified bi-dimensional axial CT images followed by a review of coronal and sagittal images. A graphical interface developed using the Visualization Toolkit (VTK) [22] is used for reconstructing and visualizing the gastric images. The tumoral lesions inspected at MSCT are located, and the morphopathological characteristics of each lesion are documented for each patient. As a result, the characteristics determined on the available images are shown.

In the second stage, the validity of the use of MSCT images such as a macroscopic technique for assessing of gastric cancer is determined. An expert physician, a stomach oncologist by means visual inspection performs the assessment. This validity 
Table 6. Original descriptive histological parameters of each TCGA-STAD patient.

\begin{tabular}{|c|c|c|c|c|c|c|c|c|c|}
\hline Case & $\mathbf{T}$ & $\mathbf{N}$ & $\mathbf{M}$ & Stage & Case & $\mathbf{T}$ & $\mathbf{N}$ & $\mathbf{M}$ & Stage \\
\hline TCGA-VQ-A8DL & T3 & N0 & M0 & IIA & TCGA-VQ-A91U & $\mathrm{T} 3$ & N1 & M0 & IIIA \\
\hline TCGA-VQ-A8DT & T3 & N3a & M0 & IIIB & TCGA-VQ-A91V & T3 & N2 & M0 & IIIA \\
\hline TCGA-VQ-A8DU & T3 & $\mathrm{N} 2$ & M0 & IIIA & TCGA-VQ-A91X & $\mathrm{T} 3$ & $\mathrm{~N} 2$ & M0 & IIIB \\
\hline TCGA-VQ-A8DZ & $\mathrm{T} 4$ & N3 & M1 & IV & TCGA-VQ-A91Y & $\mathrm{T} 4 \mathrm{a}$ & $\mathrm{N} 3 \mathrm{~b}$ & M0 & IIIC \\
\hline TCGA-VQ-A8E0 & T3 & $\mathrm{N} 2$ & M0 & IIIA & TCGA-VQ-A91Z & $\mathrm{T} 3$ & N1 & M0 & IIIA \\
\hline TCGA-VQ-A8E2 & $\mathrm{T} 3$ & $\mathrm{~N} 2$ & M0 & IIIB & TCGA-VQ-A922 & $\mathrm{T} 4$ & N1 & M0 & IV \\
\hline TCGA-VQ-A8E3 & T3 & N0 & M0 & IIA & TCGA-VQ-A92D & $\mathrm{T} 2 \mathrm{~b}$ & NO & M0 & $\mathrm{IB}$ \\
\hline TCGA-VQ-A8E7 & $\mathrm{T} 3$ & $\mathrm{~N} 1$ & M1 & IV & TCGA-VQ-A94O & $\mathrm{T} 4 \mathrm{a}$ & N3 & M0 & IIIC \\
\hline TCGA-VQ-A8P2 & $\mathrm{T} 4 \mathrm{a}$ & $\mathrm{N} 1$ & M0 & IIIA & TCGA-VQ-A94P & $\mathrm{T} 4$ & NX & MX & IV \\
\hline TCGA-VQ-A8P3 & $\mathrm{T} 4$ & $\mathrm{~N} 1$ & M0 & IIIA & TCGA-VQ-A94R & $\mathrm{T} 4 \mathrm{a}$ & $\mathrm{N} 2$ & M0 & IV \\
\hline TCGA-VQ-A8P5 & T3 & N0 & M0 & IIA & TCGA-VQ-A94T & T3 & N2 & M0 & IIIB \\
\hline TCGA-VQ-A8P8 & $\mathrm{T} 4 \mathrm{a}$ & N0 & M0 & IIB & TCGA-VQ-A94U & $\mathrm{T} 4 \mathrm{a}$ & No & M0 & IIB \\
\hline TCGA-VQ-A8PB & T3 & N0 & M0 & II & TCGA-VQ-AA64 & $\mathrm{T} 3$ & N2 & M0 & IIIB \\
\hline TCGA-VQ-A8PD & $\mathrm{T} 4 \mathrm{a}$ & N3 & M0 & IIIC & TCGA-VQ-AA68 & $\mathrm{T} 4 \mathrm{a}$ & N3 & M0 & IIIC \\
\hline TCGA-VQ-A8PE & $\mathrm{T} 3$ & N3a & M0 & IIIB & TCGA-VQ-AA69 & $\mathrm{T} 3$ & N2 & M0 & IIIA \\
\hline TCGA-VQ-A8PF & T3 & $\mathrm{N} 3 \mathrm{~b}$ & M0 & IIIB & TCGA-VQ-AA6A & $\mathrm{T} 4$ & $\mathrm{~N} 3 \mathrm{a}$ & M0 & IIIC \\
\hline TCGA-VQ-A8PK & T3 & N3a & M0 & IIIB & TCGA-VQ-AA6B & T3 & N2 & M0 & IIIB \\
\hline TCGA-VQ-A8PT & $\mathrm{T} 4 \mathrm{~b}$ & N0 & M0 & IIIB & TCGA-VQ-AA6D & $\mathrm{T} 4 \mathrm{a}$ & N1 & M0 & IIIA \\
\hline TCGA-VQ-A91A & T3 & N3a & M0 & IIIB & TCGA-VQ-AA6F & $\mathrm{T} 3$ & N1 & M0 & IIB \\
\hline TCGA-VQ-A91D & $\mathrm{T} 4 \mathrm{~b}$ & $\mathrm{~N} 2$ & M0 & IIIC & TCGA-VQ-AA6G & T3 & N0 & M0 & IIA \\
\hline TCGA-VQ-A91E & $\mathrm{T} 4 \mathrm{~b}$ & N0 & M0 & IIIB & TCGA-VQ-AA6I & $\mathrm{T} 3$ & N3 & M0 & IIB \\
\hline TCGA-VQ-A91Q & T3 & N3 & M0 & IV & TCGA-VQ-AA6J & $\mathrm{T} 4 \mathrm{a}$ & $\mathrm{N} 2$ & M0 & IIIB \\
\hline TCGA-VQ-A91S & $\mathrm{T} 4 \mathrm{~b}$ & N1 & M0 & IIIB & TCGA-VQ-AA6K & $\mathrm{T} 4 \mathrm{a}$ & $\mathrm{N} 3 \mathrm{a}$ & M0 & IIIC \\
\hline
\end{tabular}

begins with the preparation of a comparative Table of compliance with the original histological parameters of each image, provided by the datasets. Based on this table, and establishing as a gold standard the characteristics of the type of stomach cancer reported in the TCGA dataset, the diagnosis agreement is estimated using the sensitivity and specificity as standard diagnostic accuracy statistics [23-24].

The sensitivity $\left(S_{e}\right)$ and specificity $\left(S_{p}\right)$ for all the studied cases are quantified. $S_{e}$ represents the percentage of features with a condition whose are correctly identified by our framework in the MSCT images as indeed having that feature. $S_{p}$ is considered as the probability of the proposed framework of correctly identifying, solely from among images whose are known not to have a feature, all those who do indeed not have that feature.

A true positive $(T P)$ is all feature of a specific cancer type that is assessed as a feature. A true negative $(T N)$ is all non-feature of a specific cancer type that is not assessed as a feature. The false positive $(F P)$ is all non-feature of a specific cancer type that is assessed as a feature. Meanwhile, the false negative $(F N)$ is all non-feature of a specific cancer type that is not assessed as a feature.

Positive predictive value ( $P P V)$ [25] can be also used for estimating the percentage of correspondence of the macroscopic features with respect to the visualized morphopathology. $P P V$ is the probability that a certain feature of a specific cancer type (positive feature) will be verified in the abdominal CT images. This predictive value indicates the effectiveness of the proposed framework.

Negative predictive value ( $N P V)$ (25) is also considered in order to determine the effectiveness of the proposed framework, basically because such an index quantifies the probability that a certain feature of non-specific of a cancer type (negative feature) will be verified with the images.

Finally, as the stage result, the existing gap between the documented features of the cases and 
the visualization of these features in the available MSCT images is reported.

\section{RESULTS}

The results obtained from this research are in part based upon data generated by the TCGA Research Network [18]. The clinical data included in the TCGA data collection contain the information considered as gold standard and used for the diagnosis agreement.

The graphical interface developed for visualizing the information contained in the 3-D image volumes allows evaluating the MSCT capacity to effectively reflect the morphopathological features of the stomach cancer types. The macroscopic characteristics used as inputs of Table of compliance and established according our theoretical framework, correspond with location, if the adenocarcinoma is well defined or no precise and the macroscopic shape of tumor namely, polypiod, ulcerated, circumscribed, infiltrated or linitis.

The dataset TCGA-VQ-A92D is initially inspected by an expert physician for assessing the macroscopic characteristics of stomach adenocarcinoma type 1. According to its macroscopic characteristics, type 1 cancer would be the simplest to characterize. This dataset corresponds to male of 67 years old, white and still alive. The main macroscopic feature of type 1 cancer is the presence of polypoid shapes according to Borrmann and Japanese classifications (see Figures 1 and 2). The polypoids shapes can be visualized in the MSCT slices that are shown in the first row of Figure 4. This first row shows the axial, coronal and sagittal views of the 3-D volume of dataset TCGA-VQ-A92D. The second row of the Figure 4 shows a region-of-interest (ROI) [26] for each view (axial, coronal and sagittal). These ROIs have been chosen in order to magnify the image region in which the macroscopic feature can be observed in more detail.

Figure 5 shows the axial, coronal and sagittal planes of the dataset TCGA-VQ-A8DL. The objective is to locate in the anatomical planes the macroscopic features of the cancer type 2 established according to Borrmann and Japanese classifications. The ROIs chosen from the anatomical views (second row of Figure 5) are rotated with the idea of matching the stomach wall with the horizontal plane.

From the anatomical views and the chosen ROIs (Figure 5), it is simple to conclude that the tumor is circumscribed and ulcerated because the depressed region caused by the ulcer is located visually on the three anatomical views. This is the main feature of type 2 cancer. Additionally, it is observed that the adenocarcinoma has a marginal elevation at the borders, and partial diffuse dissemination especially evident in the sagittal view.

For assessing the type 3 cancer the tomographic images of dataset TCGA-VQ-A91V are used. This data corresponds a male patient of unknown race, alive and age at initial pathologic diagnosis of 68 years

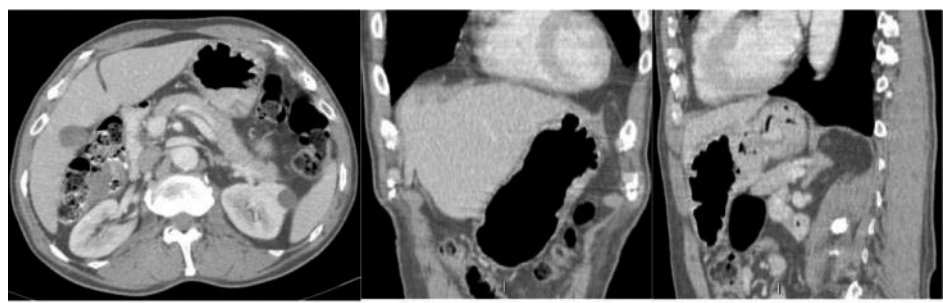

Axial View

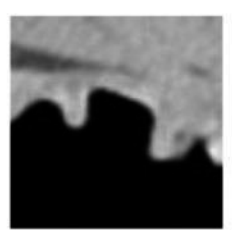

Coronal view

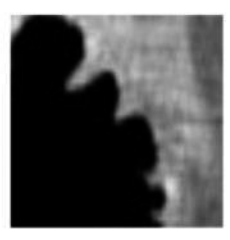

Sagittal view

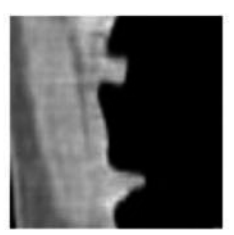

Figure 4. Results for TCGA-VQ-A92D dataset. 


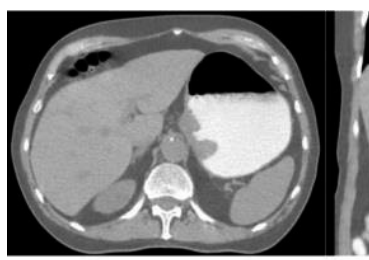

Axial View

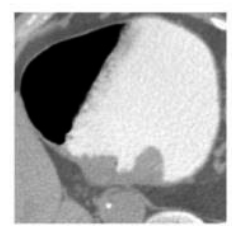

Figure 5. Results for TCGA-VQ-A8DL dataset.

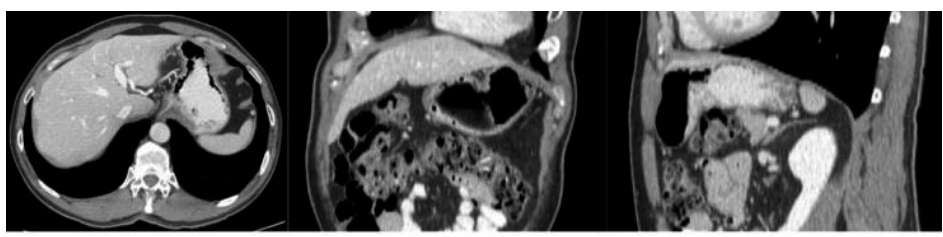

Axial View

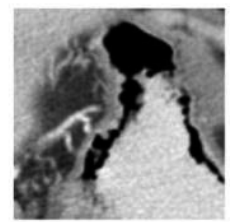

Coronal view

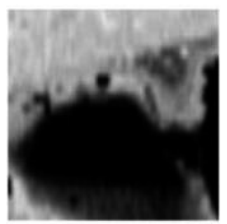

\section{Sagittal view}

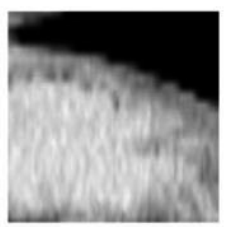

Figure 6. Results for TCGA-VQ-A91V dataset.

old. The anatomical tomographic views are shown in Figure 6. The cancer is located at the gastroesophageal (GE) junction, it is infiltrating ulcerated as can be seen in the selected ROIs (second row of Figure 6), primarily in the axial and sagittal view.

When analyzing the dataset TCGA-VQ-A94P, it is observed that the tumor is located at the fundus. Figure 7 shows the axial, coronal and sagittal tomographic views (first row), and chosen ROIs from these anatomical views (second row). Based on the clinical data documents, this is a 81 year old male patient, dead. The cancer is present in the fundus and body, rich in connective tissue and infiltrated. These features can be observed at ROIs in the anatomical views.

The comparative Table of compliance used in this research considers the inputs shown in the Table 7.
This Table shows the comparative entries only for the four datasets shown in the Figures 4-5-6-7, by effects of space in the present paper. Those four because they correspond to a patient of each type of cancer. As observed and tabulated, for these four datasets the macroscopic features analyzed can be evidenced in the tomographic images.

When performing the validation of the use of MSCT for the assessment of the type of stomach cancer in the rest of the dataset of the data collection TCGASTAD, the confidence intervals for the sensitivity and the specificity computed in order to estimate the diagnosis agreement are $[96.33 \%, 99.33 \%]$ and $[97.32 \%, 99.22 \%]$, respectively. A percentage of correspondence of the macroscopic features with respect to the visualized morphopathology is estimated from the $P P V$, in our research this value is about $96.47 \%$. Meanwhile, $N P V$ is about $99.18 \%$, 


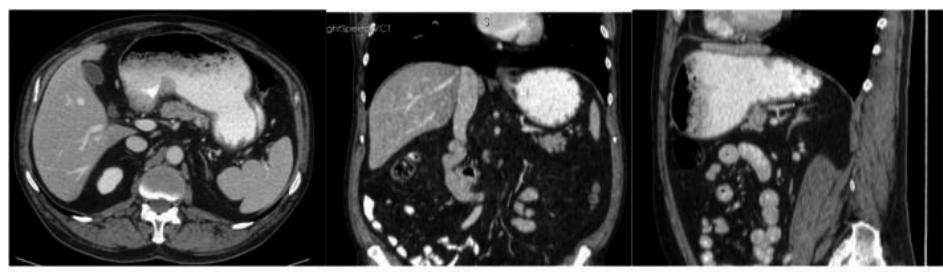

Axial View

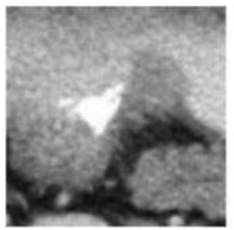

Coronal view

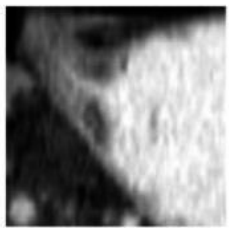

Sagittal view

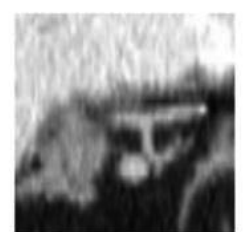

Figure 7. Results for TCGA-VQ-A94P dataset.

Table 7. Matrix of compliance for assessment of type of gastric cancer.

\begin{tabular}{|c|c|c|c|c|c|c|c|c|c|}
\hline \multirow[b]{2}{*}{ 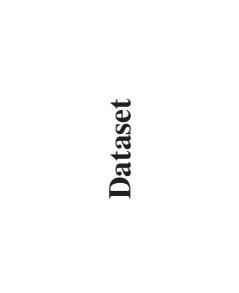 } & \multirow[b]{2}{*}{ لَّ } & \multicolumn{7}{|c|}{ Macroscopic features } & \multirow[b]{2}{*}{ હે } \\
\hline & & 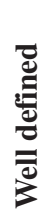 & 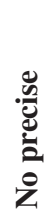 & $\frac{\overrightarrow{0}}{\stackrel{0}{0}}$ & 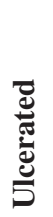 & 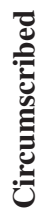 & 莺 & 昜 & \\
\hline TCGA-VQ-A92D & Proximal & $\mathrm{X}$ & & $\mathrm{X}$ & & & & & 1 \\
\hline TCGA-VQ-A8DL & Proximal & $\mathrm{X}$ & & & $\mathrm{X}$ & $\mathrm{X}$ & & & 2 \\
\hline TCGA-VQ-A91V & GE junction & & $\mathrm{X}$ & & $\mathrm{X}$ & & $\mathrm{X}$ & & 3 \\
\hline TCGA-VQ-A94P & Fundus/body & & $\mathrm{X}$ & & $\mathrm{X}$ & & & $X$ & 4 \\
\hline
\end{tabular}

which indicates that the theoretical framework based on the Borrmann classification and the Japanese gastric cancer classification proposed for assessing the stomach cancer from MSCT images presents a high percentage of assessment of non-specific characteristics for a specific cancer type.

\section{DISCUSSION}

In two of the datasets contained in TCGA-STAD, the macroscopic features observed in the MSCT slices (including all anatomical views) are not matched with the type of cancer documented in the clinical data of each dataset. In both cases, certain macroscopic features are verified which do not correspond to the histologically assigned typification. In this sense, in about $96 \%$ of inspecting images, the visual characteristics and the theoretical characteristics are matched. For example, for type 3 cancer with features such as ulcerated, noncircumscribed, possibly with polypiod components and infiltration, the visual inspection of axial, coronal and sagittal views evidence the circumscribed shape of tumor. Secondly, for example, for type 4 cancer the visual inspection determines that it is non-diffuse.

In the dataset TCGA, no patients with cancer type 0 and 5 were verified. However, according to the Japanese classification, it is considered difficult to classify the cancer type 0 -I and type 0 -II because they could be confused with types 1 and 2 of the Borrmann classification. It would require the very accurate measurement of the size of the protuberance and the excavation, respectively. The same difficulty would be with the subclassifications of type 0-II cancer.

\section{CONCLUSIONS}

A two-phase procedure has been developed for validating the effectiveness (predictive capacity) of computed tomography for replicating the detection 
parameters, theoretically established, of types of gastric adenocarcinoma.

The procedure is applied to 6210 tomography slices from 46 MSCT explorations acquired of patients diagnosed, from the cancer data collection of the project, Cancer Genome Atlas Stomach Adenocarcinoma (TCGA-STAD).

A comparative analysis based on the document review and direct observation that uses a matrix of compliance with the specific qualities of the theoretical standards, in the visualization of images performed by the clinical specialist from the datasets is performed to validate the effectiveness of computed tomography for replicating the macroscopic features of each type of stomach cancer.

From this analysis, the general conclusion is that the computed tomography analyzed by a clinical specialist is a precise imaging modality in the macroscopy qualitative assessment of the staging of stomach cancer.

Despite the highly favorable result obtained in this research, the MSCT, as the theory indicates, is a more confirmatory technique than a preliminary diagnostic technique for gastric cancer prognosis. The triangulation [27] of the qualitative information extracted from the MSCT medical images and the quantitative histological information is required to give conclusive evidence about the type of adenocarcinoma of stomach. The macroscopic analysis technique would become a more exploratory diagnosis if there were automatic computerized systems for exploration and assessment of the amount of data that are present in the images of MSCT more efficient, workable and effective.

In the Research Group of the authors, there is an orientation to the design of algorithms for the automatic assessment of the morphopathology of certain anatomical structures through medical images, but in the diagnostic processes, prior to the development of the research, it has been concluded that it is highly necessary to be clear about all the macroscopic morphological characteristics that can be observed and obviously detected from the images. Meanwhile, the experience that the Group clinician (BV) has in the area of stomach cancer diagnosis to advance in the knowledge of these characteristics was taken advantage of. The main problem is that there is a single associated specialist. However, through the medical academic program, a recruitment process has been undertaken by oncology specialization students who join and train with the help of this procedure. As regards the development of automated systems, progress has been performed in the detection and assessment of type 2 cancer [28].

\section{ACKNOWLEDGEMENTS}

Authors would like to thank the Universidad Simón Bolívar, Colombia, and Investigation Dean's Office of Universidad Nacional Experimental del Táchira, Venezuela for their support to this research. This work was supported by the Universidad Simón Bolívar, Colombia (grant: C2011720117).

\section{REFERENCES}

[1] L.H. Sobin. "International histological classification of tumors". Second Edition. Cancer. Vol. 63, Issue 5, pp. 907-907. 1989.

[2] A. DiMarino and S. Benjamin. "Gastrointestinal Disease: An Endoscopic Approach”. Slack. 2002.

[3] A. Fritz, C. Percy, A. Jack, K. Shanmugaratnam, L. Sobin and D.M.P.S. Whelan. "International Classification of Diseases for Oncology". World Health Organization, third edition. 2012.

[4] S. Edge, D. Byrd, C. Compton, A. Fritz, F. Greene and A. Trotti. "AJCC Cancer Staging Handbook". Springer-Verlag, New York, 7th edition. 2010.

[5] S. Edge and C. Compton. "The American Joint Committee on Cancer: the 7th Edition of the AJCC Cancer Staging Manual and the Future of TNM". Annals of Surgical Oncology. Vol. 17, Issue 6, pp. 1471-1474. 2010. DOI: 10.1245/s10434-010-0985-4.

[6] F.L. Greene. "TNM: Our language of cancer". CA: A Cancer Journal for Clinicians. Vol. 54, Issue 3, pp. 129-130. 2004. DOI: 10.3322/ canjclin.54.3.129.

[7] R. Borrmann. "Geschwulste des margens". In F. Henke and O. Lubarsch, editors, Handbuch spez pathol anat und hist. Springer-Verlag, pp. 864-871. 1926.

[8] Japanese Gastric Cancer Association. "Japanese classification of gastric carcinoma". 
3rd English edition. Gastric Cancer. Vol. 14, Issue 2, pp. 101-112. 2011. DOI: 10.1007/ s10120-011-0041-5.

[9] P. Rutkowski, A. Wozniak, M. Debiec-Rychter, M. Kakol, W. Dziewirski, M. Zdzienicki, K. Ptaszynski, M. Jurkowska, J. Limon and J.A. Siedlecki. "Clinical utility of the new American Joint Committee on Cancer staging system for gastrointestinal stromal tumors". Cancer. Vol. 117, Issue 21, pp. 4916-4924. 2011. DOI: $10.1002 /$ cncr.26079.

[10] T. Kajitani. "The general rules for the gastric cancer study insurgery and pathology". The Japanese journal of surgery. Vol. 11, Issue 2, pp. 127-139. 1981. DOI: 10.1007/ BF02468883.

[11] D. Day, J. Jass, A. Price, N. Shepherd, J. Sloan, N. Talbot, G. Williams and B. Warren. "Morson and Dawson's Gastrointestinal Pathology". Wiley. 2008.

[12] R.A. Robb and M.L. Morin. "Principles and instrumentation for dynamic X-ray computed tomography". In: M. Marcus, H. Schelbert, D. Skorton, G. Wolf editors: Cardiac Imaging. A Companion to Braunwald's Heart Disease. Philadelphia: W.B. Saunders Company, pp. 634-668. 1991.

[13] H. Hu. "Multi-slice helical CT: Scan and reconstruction". Medical Physics. Vol. 26, Issue 1, pp. 5-18. 1999.

[14] R.H. de O. Barros, T.J. Penachim, D.L. Martins, N.A. Andreollo and N.M.G. Caserta. "Multidetector computed tomography in the preoperative staging of gastric adenocarcinoma". Radiologia Brasileira. Vol. 48, Issue 2, pp. 74-80. 2015.

[15] K.M. Horton and E.K. Fishman. "Current role of CT in imaging of the stomach". Radiographics. Vol. 23, Issue 1, pp. 75-87. 2003.

[16] J.S. Lim, M.J. Yun, M.-J. Kim, W.J. Hyung, M.-S. Park, J.-Y. Choi, T.-S. Kim, J.D. Lee, S.H. Noh and K.W. Kim. "CT and PET in stomach cancer preoperative staging and monitoring of response to therapy". RadioGraphics. Vol. 26, Issue 1, pp. 143-156. 2006.

[17] K. Yao. "The endoscopic diagnosis of early gastric cancer". Annals of Gastroenterology. Vol. 26, Issue 1, pp. 11-22. 2013.

[18] F.R. Lucchesi and N.D. Aredes. "Radiology Data from The Cancer Genome Atlas Stomach Adenocarcinoma". The Cancer Imaging
Archive, TCGA-STAD collection. 2016. URL: http://doi.org/10.7937/K9/TCIA.2016. GDHL9KIM

[19] K. Clark, B. Vendt, K. Smith, J. Freymann, J. Kirby, P. Koppel, S. Moore, S. Phillips, D. Maffitt, M. Pringle, L. Tarbox and F. Prior. "The Cancer Imaging Archive (TCIA): Maintaining and Operating a Public Information Repository". Journal of Digital Imaging. Vol. 26, Issue 6, pp. 1045-1057. 2013. DOI:10.1007/s10278-013-9622-7.

[20] C.C. Jaffe. "Imaging and genomics: Is there a synergy?". Radiology. Vol. 264, Issue 2, pp. 329-331. 2012. DOI: 10.1148/ radiol.12120871.

[21] A. Holmes. "Direct observation". In F.R. Volkmar editor, Encyclopedia of Autism Spectrum Disorders, pp. 980-981. Springer, New York. 2013.

[22] W. Schroeder. "The visualization toolkit: an object-oriented approach to 3D graphics". Kitware, Clifton Park, N.Y. 2006.

[23] M.S. Pepe. "The Statistical Evaluation of Medical Tests for Classification and Prediction". New York: Oxford University Press. 2003.

[24] H.A. Katki, Y. Li, D.W. Edelstein and P.E. Castle. "Estimating the agreement and diagnostic accuracy of two diagnostic tests when one test is conducted on only a subsample of specimens". Statistics in Medicine. Vol. 31, Issue 5. 2012.

[25] R. Trevethan. "Sensitivity, Specificity and Predictive Values: Foundations, Pliabilities, and Pitfalls in Research and Practice". Frontiers in Public Health $N^{\circ}$ 5. 2017.

[26] R. Brinkmann. "The Art and Science of Digital Compositing”. Computer graphics/ Multimedia. Morgan Kaufmann. 1999.

[27] J. Creswell and J. Creswell. "Research Design: Qualitative, Quantitative and Mixed Methods Approaches". SAGE Publications. 2017.

[28] G. Chacón, J. Rodríguez, V. Bermúdez, M. Vera, J. Hernández, S. Vargas, A. Pardo, C. Lameda, D. Madriz and A. Bravo. "Computational assessment of stomach tumor volume from Multi-Slice Computerized Tomography images in presence of type 2 cancer", F1000research. Vol. 7, Issue 1098. 2018. DOI: 10.12688/f1000research.14491.2. 